\title{
High resolution SMA imaging of (ultra)-luminous infrared galaxies
}

\author{
Qinghua Tan ${ }^{1,2,3}$, Yu Gao ${ }^{1,2}$, Zhong Wang ${ }^{4}$, and Vivian $\mathbf{U}^{4,5}$ \\ ${ }^{1}$ Purple Mountain Observatory, Chinese Academy of Sciences, Nanjing 210008, China \\ ${ }^{2}$ Key Laboratory of Radio Astronomy, Chinese Academy of Sciences, Nanjing 210008, China \\ ${ }^{3}$ Graduate School of CAS, Beijing 100039, China \\ ${ }^{4}$ Harvard-Smithsonian Center for Astrophysics, Cambridge, MA02138, USA \\ ${ }^{5}$ Institute for Astronomy, University of Hawaii, 2680 Woodlawn Drive, Honolulu, HI 96822,
}

USA

\begin{abstract}
We present preliminary results on Submillimeter Array (SMA) observations of three Luminous Infrared Galaxies (LIRGs) and one infrared Quasi Stellar Object (IR QSO). The galaxies were observed at sub-kpc spatial resolution in lines of $\mathrm{CO}$ and ${ }^{13} \mathrm{CO}$, as well as in the continuum at $1.3 \mathrm{~mm}$. The moment maps show that the molecular gas in these galaxies is distributed in rotating disks with velocity gradients ranging from $\sim 30$ to $120 \mathrm{~km} \mathrm{~s}^{-1} \mathrm{kpc}^{-1}$. Combined with archival CO $\mathrm{J}=3-2$ data, the spatial distributions of the CO $J=3-2 / J=2-1$ ratios shows clear variations across the galaxies. The brightness temperature of the overlap region in VV 114 is found to be lower than that in the nuclear region of VV 114E, suggesting that that the bulk of molecular gas in this region is sub-thermalized.
\end{abstract}

Keywords. galaxies: individual (VV114, NGC 1614, IRAS 17578-0400, IRAS F22454-1744) galaxies: interactions - galaxies: starburst

\section{Introduction}

Strong interactions or mergers between gas-rich disk galaxies is considered to be one of the primary mechanisms to trigger the intense star formation in luminous infrared galaxies (Sanders \& Mirabel 1996). Tidal interaction between progenitor galaxies will transport the gas to the nucleus, condense the gas and trigger a widespread starburst. Since the proposal that ULIRGs hosting an AGN are transition objects that eventually evolve into optical QSOs (Sanders et al. 1988), more recent studies have focused on the evolutionary connection (Hopkins et al. 2006). However, for the galaxies at high redshifts, two mechanisms, major merger and cold mode accretion, have been suggested as drivers of active star formation (Narayanan et al. 2010; Keres et al. 2005). Thus, systematic studies of local luminous infrared galaxies are essential for the understanding of galaxy evolution.

Because molecular gas provides fuel for star formation and can carry the shocks, transporting the angular momentum during the galaxy interaction, the physical properties, distribution and kinematics of molecular gas are important for understanding the physical processes in galaxy mergers. Massive molecular gas reservoirs in (U)LIRGs have been revealed in previous studies and high resolution observations show that a substantial fraction of molecular gas lies within their central kpc regions (Downes \& Solomon 1998; Wilson et al. 2008). Therefore, together with multiwavelength imaging studies, high resolution mapping of molecular gas in a large sample of (U)LIRGs will help provide a comprehensive picture of star formation activity during the merging process. 
Table 1. Summary of basic properties

\begin{tabular}{lccccccl}
\hline Galaxy & $\begin{array}{c}c z \\
\left(\mathrm{~km} \mathrm{~s}^{-1}\right)\end{array}$ & $\begin{array}{c}\log L_{\mathrm{FIR}} \\
\left(L_{\odot}\right)\end{array}$ & $\begin{array}{c}\text { Array } \\
\text { Config. }\end{array}$ & $\begin{array}{c}\text { Freq. } \\
(\mathrm{GHz})\end{array}$ & $\begin{array}{c}\text { Beam Size } \\
(\operatorname{arcsec})\end{array}$ & $\begin{array}{c}\text { Spatial Scale } \\
\left(\mathrm{kpc} / 1^{\prime \prime}\right)\end{array}$ & $\begin{array}{l}\text { Vel. Gradient } \\
\left(\mathrm{km} \mathrm{s}^{-1} \mathrm{kpc}^{-1}\right)\end{array}$ \\
\hline VV 114 & 6016 & 11.71 & Extended & 230 & $1.52 \times 1.36$ & 0.40 & $100^{1}$ \\
NGC 1614 & 4778 & 11.65 & Extended & 230 & $1.22 \times 1.06$ & 0.32 & 120 \\
IRAS 17578-0400 & 4210 & 11.48 & Compact & 230 & $3.09 \times 2.50$ & 0.28 & 50 \\
IRAS F22454-1744 & 35166 & 12.13 & Extended & 230 & $1.81 \times 1.19$ & 2.10 & 30 \\
\hline
\end{tabular}

${ }^{1}$ Velocity gradient along the north-south direction.

In this paper, we present new data obtained with the SMA for the first four galaxies in the $\mathrm{CO} J=2-1$ line. In addition, we present preliminary results on the CO $J=3-2 / J=2-1$ line ratio distribution by combining the new data with archival CO $J=3-2$ data.

\section{Observations and Data reduction}

Our data were taken with the SMA between 2009 August and 2010 May. Each galaxy was observed in $\mathrm{CO}$ and ${ }^{13} \mathrm{CO}$ lines and in the $1.3 \mathrm{~mm}$ continuum. The HPBW of the primary beam is $\sim 52^{\prime \prime}$ at these observing frequencies. The array configuration and synthesized beam size for each galaxy are listed in Table 1.

The initial data calibration was carried out using the MIR software package. Observations of planets or moons such as Uranus and Callisto were taken for determining the flux of the gain calibrator, which is a nearby quasar observed every $\sim 20$ mins to calibrate phase gain variations with time. Bandpass calibration was determined using a strong quasar. The further editing and imaging were done using the MIRIAD package.

\section{Results}

The high resolution CO $J=2-1$ integrated intensity, velocity field and dispersion maps for the four galaxies observed with the SMA are shown in Figure 1. The CO emission was found to be distributed over the central 2-6 kpc region of the rotating disk, with peak velocity dispersion ranging from 150 to $250 \mathrm{~km} \mathrm{~s}^{-1}$. On a linear scale, the observed velocity gradient over the $\mathrm{CO}$ emission region is between 30 and $120 \mathrm{~km} \mathrm{~s}^{-1} \mathrm{kpc}^{-1}$. The global average line ratio of $\mathrm{CO} /{ }^{13} \mathrm{CO} J=2-1$ for the three galaxies with ${ }^{13} \mathrm{CO}$ detection is $\sim 25$, which is comparable to the line ratio of $\mathrm{CO} /{ }^{13} \mathrm{CO} J=1-0$ found in LIRGs (Aalto et al. 1991).

For VV 114, the significantly improved high resolution CO $J=2-1$ image shows a barlike structure with two peaks and a molecular tail, similar to that seen in CO $J=3-2$ (Iono et al. 2004). The irregular velocity field appears to reveal two distinct kinematical components with major axes along the north-south and east-west directions respectively. The CO map of IRAS 17578-0400 exhibits two emission peaks. In the extension to the northwest, the velocity contours are more irregular, perhaps caused by an additional kinematical component. Both the position-velocity diagram along the major axis and the channel map show that the secondary peak in the southeast is a single component slightly offset in the position, and based on the optical image which also exhibits two peaks, we consider the secondary CO peak might come from a separate physical component. For the luminous infrared QSO IRAS F22454-1744, the gaussian fit to the image implies a slightly resolved source, with a deconvolved source size of FWHM $=3.1^{\prime \prime} \times 1.3^{\prime \prime}$, corresponding to an average radius of $\sim 2.4 \mathrm{kpc}$. Both the extended gas distribution and the ordered velocity gradient found in moment maps seems to suggest a rotating 

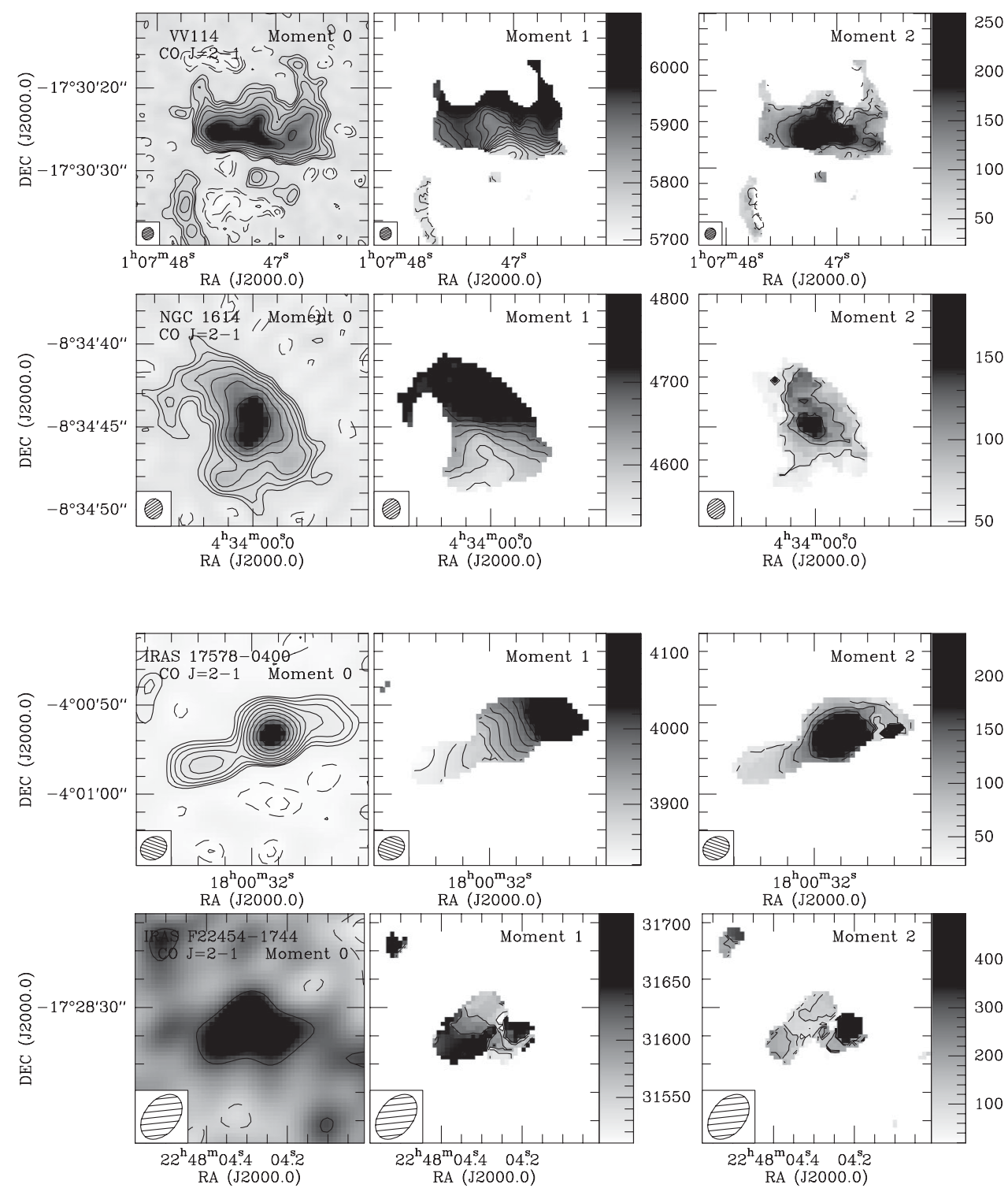

Figure 1. SMA CO $J=2-1$ moment maps. The lowest contour of moment 0 map is $\pm 2 \sigma$ and contours increase by factors of 1.5 (except IRAS F22454 with contour increase in steps of $1 \sigma$ ). The moment 1 and 2 maps were made using the positive signal $>3 \sigma$ and blanked where the integrated intensity is $<2 \sigma$. The synthesized beam size for each map is listed in Table 1 . The unit of the colorbar is $\mathrm{km} \mathrm{s}^{-1}$. For VV 114 and NGC 1614, the moment maps were derived from the combination of Extended and Compact data.

disk structure. However, observations with higher resolution and sensitivity are urgently needed for further confirmation.

Combined with the archival CO $J=3-2$ data (Wilson et al. 2008), we convolved the CO $J=2-1$ emission to the resolution of the $J=3-2$ image (Fig. 2). The line ratio map of CO $J=3-2 / J=2-1$ clearly shows that the gas temperature varies across the galaxy. The overlap region of VV 114 exhibits lower line ratios than those measured towards the nuclear region in VV 114E, suggesting that cold and extensive molecular gas are 


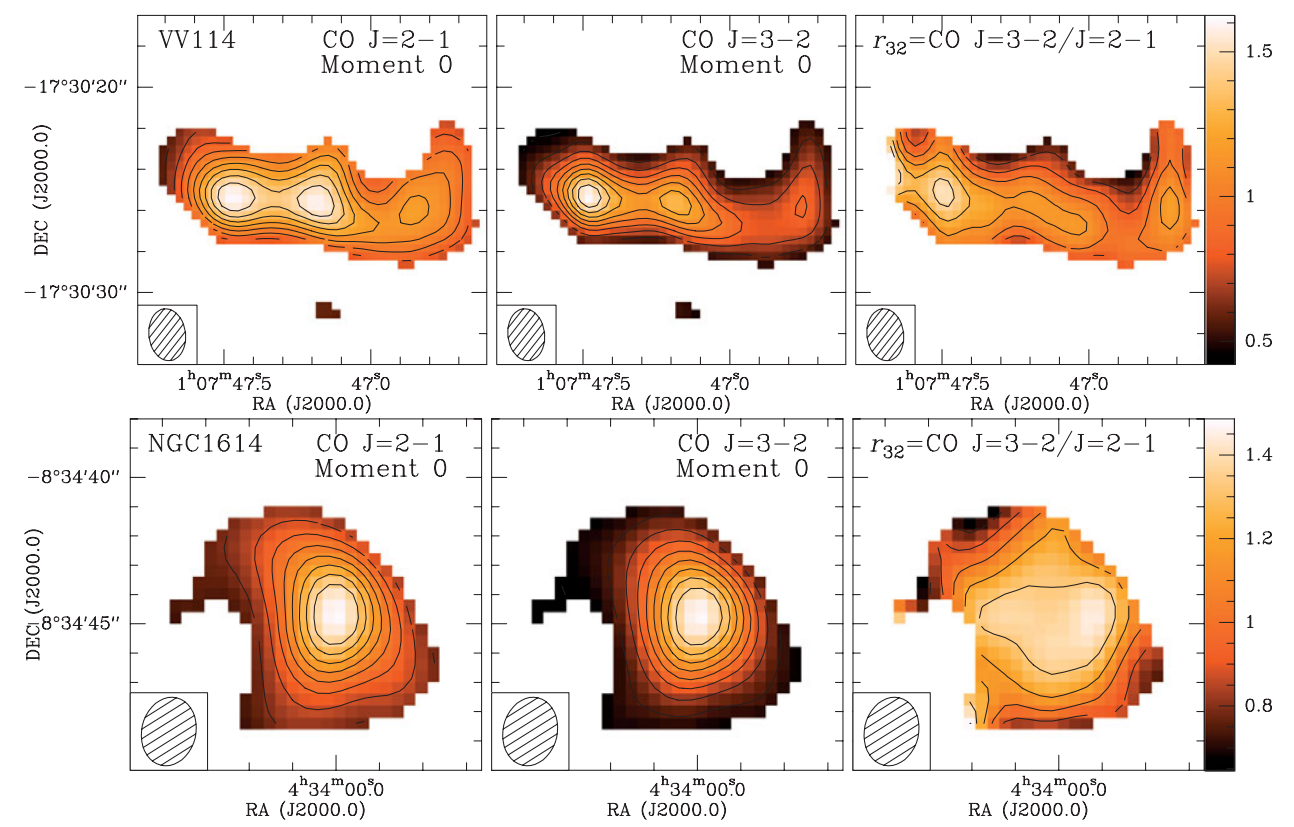

Figure 2. CO $J=2-1, J=3-2$ and $J=3-2 / J=2-1$ line ratio maps where both measurements are above $3 \sigma$. The CO $J=2-1$ maps have been convolved to the resolution of the $J=3-2$ maps. The synthesized beam sizes of the CO $J=3-2$ maps for VV 114 and NGC 1614 are $2.54^{\prime \prime} \times 1.79^{\prime \prime}$ and $2.36^{\prime \prime} \times 1.85^{\prime \prime}$, respectively

predominant in this region. Taking the gas temperature distribution and the largest velocity dispersion found in the overlap region (Fig. 1) into consideration, this result is likely to indicate that a significant amount of molecular gas is flowing into this region and possibly forming the new dynamical center of the system (Yun et al. 1994). For NGC 1614, the line ratio has a value above unity, and varies little across the galaxy, implying that the majority of the molecular gas is fully thermalized.

\section{References}

Aalto, S., Black, J., Johansson, L., \& Booth, R. 1991, A\& A, 249, 323

Downes, D. \& Solomon, P. 1998, ApJ, 507, 615

Keres, D., Katz, N., Weinberg, D., et al. 2005, MNRAS, 363, 2

Hopkins, P., Somerville, R., Hernquist, L., et al. 2006, ApJ, 652, 864

Iono, D., Ho, P., Yun, M., et al. 2004, ApJ(Letters), 616, L63

Narayanan, D., Hayward, C., Cox, T., et al. 2010, MNRAS, 401, 1613

Sanders, D., Soifer, B., Elias, J., Neugebauer, G., \& Matthews, K. 1988, ApJ(Letters), 328, L35

Sanders, D. \& Mirabel, I. 1996, ARAA, 34, 749

Wilson, C., Petitpas, G., Iono, D., et al. 2008, ApJ, 178, 189

Yun, M., Scoville, N., \& Knop, R. 1994, ApJ(Letters), 430, L109 